\title{
The anti-symmetry principle for quasi-static crack propagation in Mode III.
}

\author{
Gerardo E. Oleaga \\ June 1, 2007 \\ Departamento de Matemática Aplicada \\ Facultad de Matemáticas \\ Universidad Complutense de Madrid \\ 28040 Madrid \\ Spain.
}

\begin{abstract}
In this note we study a basic propagation criterion for quasi-static crack evolution in Mode III. Using classical techniques of complex analysis, the assumption of stable growth is expressed in terms of the parameters defining the elastic field around the tip. We explore the consequences of the local condition obtained and analyse its role as a crack propagation law. In particular, we herein extend to bounded domains a number of results previously obtained for the whole plane.
\end{abstract}

\section{Introduction}

The present work deals with some fundamental questions in linear elastic fracture mechanics in its simplest framework; namely, out of plane fields, brittle homogeneous materials, quasistatic propagation and Griffith's dissipation model. The starting point is the following basic question: how the shape of a growing crack is determined by the local field around the tip? This problem is intimately related to that of finding a suitable propagation criterion. There is a huge amount of literature about this subject, and we shortly review a few previous contributions to establish a reference for the forthcoming discussion.

To our knowledge, one of the most widely accepted criteria is the so-called principle of local symmetry. It was proposed by Goldstein and Salganik in [7] and later analysed by Cotterell and Rice in [3]. For quasi-static propagation in a two dimensional in-plane elastic 
field, it can be formulated as follows: the crack grows along the path that cancels the Mode II stress intensity factor, so that:

$$
K_{I I}=0
$$

This equation is added to the energy conservation law, expressed in terms of the stress intensity factors through the celebrated Griffith-Irwin relationship:

$$
\frac{1}{Y}\left(K_{I}^{2}+K_{I I}^{2}\right)=\kappa
$$

Here $\kappa$ is a material constant defining the amount of energy per unit length that has to be provided to open the crack; $Y$ is the Young modulus. Both scalar equations provide conditions linking the path shape and the applied loads through the parameters defining the strength of the field near the tip; a criterion with this property is therefore termed local. Notice that the left hand side of (2) represents the so-called energy release rate for a crack propagating smoothly, ie. without kinking or branching. The principle completes the mathematical formulation of the free boundary problem, since we have two scalar equations to find the evolution of a plane curve. Nevertheless, it is not physically complete, because we are imposing the artificial constraint of path smoothness.

Another well-known local criterion is the maximum energy release rate principle. In our view, it is the most straightforward way to approach the problem from basic physical principles. Consider a family of virtual extensions $\gamma_{\alpha}(t)$ of the actual crack configuration. In this setting, $t \geq 0$ is a parametrisation of the extended curve, $\alpha$ defines the specific curve of the family and $\gamma_{\alpha}(t)$ represents the tip of the extended crack at "time" $t$. Let $\Delta E(t ; \alpha)$ denote the amount of mechanical energy released along this virtual extension and $\Delta Q(t ; \alpha)$ the amount of dissipation involved (ie. minus the work that must be done to break the atom bonds on crack faces). We can say that the evolution along the path $\Delta \Gamma_{\alpha}:=\left\{\gamma_{\alpha}(t), t \geq 0\right\}$ is possible if the following inequality holds for some small enough $\delta>0$ :

$$
-\Delta E(t ; \alpha) \geq \Delta Q(t ; \alpha) \quad \forall t \leq \delta
$$

If we have a strict inequality in (3), the crack evolution turns to be unstable, and other physical ingredients should enter the picture, for instance the kinetic energy flux. On the other hand, we say that a crack configuration is stable if the following condition holds: "For all virtual extensions $\Delta \Gamma_{\alpha}$, there exists some $\delta>0$ such that:

$$
-\Delta E(t ; \alpha) \leq \Delta Q(t ; \alpha) \text { for all } t<\delta . "
$$

Consider now two cases of this inequality. For a finite extension, $\Delta Q(t ; \alpha)$ is always greater than zero (we have to do negative mechanical work to break the bonds on crack faces). On the other hand, $\Delta E$ is always negative (elastic energy decreases with crack growth). We consider the following limit for a fixed $\alpha$ :

$$
L(\alpha):=\lim _{t \rightarrow 0}\left(-\frac{\Delta E(t ; \alpha)}{\Delta Q(t ; \alpha)}\right) .
$$


If $L(\alpha)$ is strictly greater than 1 , according to (3) unstable growth along this path will take place. On the other hand, if it is strictly lower than 1 we say that crack growth along this way is not possible. According to this, we say that the curve evolves in a quasi-static or critical regime if it satisfies the stability condition (4) and at each configuration there exists some $\alpha$ with $L(\alpha)=1$ (critical growth).

When Griffith's model enters the picture, the dissipative term is proportional to the length of the extended crack, that is $\Delta Q(t ; \alpha)=\kappa l(t)$. Therefore, the limit in (5) is written as:

$$
L(\alpha)=\lim _{t \rightarrow 0}\left(-\frac{\Delta E(t ; \alpha)}{\kappa l(t)}\right)=: \frac{1}{\kappa} G(\alpha),
$$

where $G(\alpha)$ is the energy release rate. In order to check that the critical growth condition is satisfied, we must ensure first that there are no possible unstable directions along other paths. This leads us to the following inequality:

$$
G(\alpha) \leq \kappa
$$

Then, if a crack propagates under a critical or quasi-static regime, the growth directions are defined as follows:

$$
\alpha^{*}=\text { crack growth direction } \Rightarrow G\left(\alpha^{*}\right)=\max _{\alpha} G(\alpha) .
$$

This is the so-called maximum energy release rate criterion.

In this note we start by reviewing the role of the configurational force (6) as a basic object to determine the path of a quasi-static growing crack in Mode III. In Section 2 we show that this concept alone is not enough to define the shape evolution in an out of plane setting and a more precise study of $\Delta E$ should be done. In Sections 3 and 4 we resume the picture presented in our previous work [14, 15], where a basic condition for crack stability called the anti-symmetry principle was obtained for an unbounded domain, and give some further insight about the kind of crack propagation law obtained. In Section 5 we analyse the case of a bounded domain, not considered in [14, 15], and the main differences with the former one are pointed out.

\section{The role of the driving force in crack direction.}

The discussion in the previous Section indicates that the function $G(\alpha)$ in (6) has to be computed in the first place. This task was performed by several authors and it is worth to review some of these works here. Let us parametrise the crack growing direction by the number $\alpha:=\varphi / \pi$, where $\varphi$ is the kinking angle with respect to the initial crack. Consider first some theoretical formulae for this quantity. In the analysis of the energy released for a virtual crack path, we have the well known Eshelby-Rice-Cherepanov $J$ integral for straight extensions (cf. [16]). Explicitly, for $\alpha=0$ we have that:

$$
G(0)=J_{1}
$$


where $J_{1}$ (formula (68) in [16]) is the component, in the direction parallel to the existing crack, of the vector given by (summation on repeated indices is assumed):

$$
J_{k}:=\int_{C}\left(U n_{k}-\sigma_{i j} u_{i, j} n_{j}\right) d s
$$

where $|\vec{n}|=1$ is normal to $C$ (a Jordan curve surrounding the crack tip), $U$ is the elastic energy density, $\sigma_{i j}$ are the components of the stress tensor and $u_{i}$ is the displacement field. In pure Mode III, (10) reduces to the expression:

$$
J_{k}=\int_{C}\left(U n_{k}-\sigma_{3 j} u_{3, j} n_{j}\right) d s \quad(j, k=1,2) .
$$

Thus, $J_{1}$ gives the energy release rate for a straight extension of the preexisting crack. Nevertheless, (9) tells us nothing for other virtual directions of propagation. It should be pointed out that in Rice's article, the quantity $J$ is not considered as a vectorial force.

More recently, Gurtin introduced the vector (10) in [8, formula (4.6)] with a different notation. This may be considered as a natural generalization of (9) when the coordinate axes are not parallel to the crack direction. The formula for $G$ turns to be:

$$
G=\vec{J} \cdot \vec{e},
$$

where $\vec{e}$ is a unit vector in the direction of (quasi-static) motion. This expression suggests that $\vec{J}$ is a mechanical force yielding the energy released for arbitrary directions of motion, while freezing the actual configuration. But this is not the case, since (11) is only valid for smooth extensions of the initial crack. Then it is not possible to give $\vec{J}$ the status of a force as a "gradient" of some free energy.

Let us see that (11) gives a wrong answer when $\vec{e}$ is not pointing in the direction of the current crack configuration. For this kind of "kinked" paths in Mode III the stress intensity factor was obtained by Sih [17] applying uniform loads at infinity. By the well-known Griffith-Irwin relationship, we can apply this result to compute the energy release rate for different directions:

$$
G(\alpha)=\frac{K^{2}}{2 \mu}\left(\frac{1-\alpha}{1+\alpha}\right)^{\alpha} \quad \alpha:=\varphi / \pi
$$

$K$ being the stress intensity factor for the initial configuration, and $\mu$ the shear modulus. In Figure 1 we see a comparison between the true energy release rate and the one obtained using $\vec{J}$ as a generalised force in (11).

It is to be noted that, even if $\vec{J}$ cannot be used as a good reference for the work done by the elastic forces, it shows correctly that the best direction to release energy is the same as the one defined by the preexisting crack (for Mode III). We conclude that the "crack driving force", or more precisely the first order variation of the energy, is not enough to determine the crack geometry: any smooth curve is compatible with the condition of maximum $G$ 


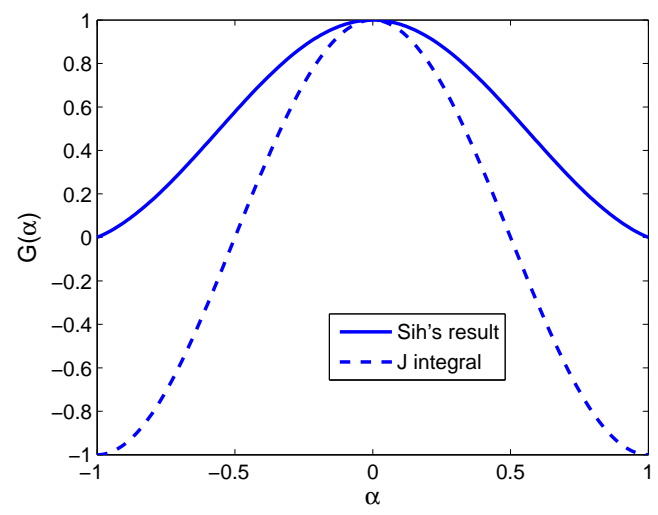

Figure 1: The energy release rate for different growing directions.

imposed in (8). On the other hand, in addition to the well-known Griffith's balance during critical growth

$$
G(0)=\kappa
$$

it is certainly useful to establish a necessary condition for the stability of the crack configuration.

\section{The energy functional for kinks and straight extensions}

In the previous Section we showed that in Mode III we should study the energy released functional in more detail to find a complete crack evolution law from first principles. We will now summarize the results obtained in [14] where the energy released for a finite extension of the crack is expanded in terms of crack length and more information about crack direction is obtained. We refer to that article for any technical detailed not included here.

\subsection{The boundary value problem}

The energy release rate for different growing directions, shown in (12), was obtained in [17] for an initially rectilinear crack in the plane, subjected to uniform loading at infinity. This boundary conditions define a local field that is probably not rich enough to capture the growing directional preference. To overcome this (possible) drawback, we considered the semi-infinite straight crack as initial configuration with an arbitrary displacement field $u_{0}$ around it, satisfying the equilibrium equation with no tractions on $\Gamma_{0}$ :

$$
\begin{aligned}
\Delta u_{0}=0 & \text { in } \mathbb{R}^{2} \backslash \Gamma_{0}, \\
\partial_{n} u_{0}=0 & \text { on } \Gamma_{0} .
\end{aligned}
$$


We also add the finite energy condition around the tip, for some $r>0$ :

$$
\int_{B_{r}(\operatorname{tip})}\left|\nabla u_{0}\right|^{2}<\infty
$$

Once the crack is extended, similar conditions hold for $u_{l}$ together with the "matching condition":

$$
\left|u_{l}-u_{0}\right| \rightarrow 0 \quad \text { uniformly at infinity }
$$
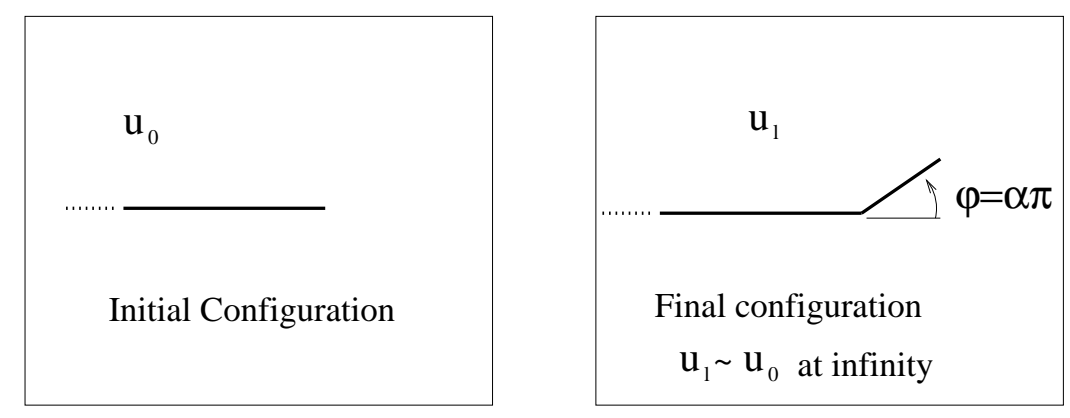

Figure 2: Problem setting

Notice that in this case we are using $t \equiv l=$ length of the crack extension to parametrise the displacements. In this way, the elastic field at infinity is fixed during crack advance and there is no mechanical work of the applied loading. The function $u_{l}$ satisfies a mixed boundary value problem and it is well defined for a given initial field and crack configuration $\Gamma_{l}$ (cf. [14] for the details).

Using basic properties of harmonic functions we write the field as the real part of an analytic function $\eta_{0}$ :

$$
u_{0}=\operatorname{Re}[\eta(\zeta)] \quad \eta_{0} \text { analytic in } \mathbb{C} \backslash \Gamma_{0} .
$$

Taking into account the assumed Neumann homogeneous condition, it follows that $\eta_{0}^{\prime}$ is such that:

$$
\eta_{0}^{\prime}(\zeta)=\frac{\partial u_{0}}{\partial x}-i \frac{\partial u_{0}}{\partial y} \Rightarrow \eta_{0}^{\prime} \text { real on } \Gamma_{0}
$$

The initial configuration may be carried to the upper half plane by the elementary map

$$
f_{0}(z)=-z^{2}
$$

Consequently, the complex function given by

$$
h_{0}(z):=\eta_{0}\left(f_{0}(z)\right)
$$




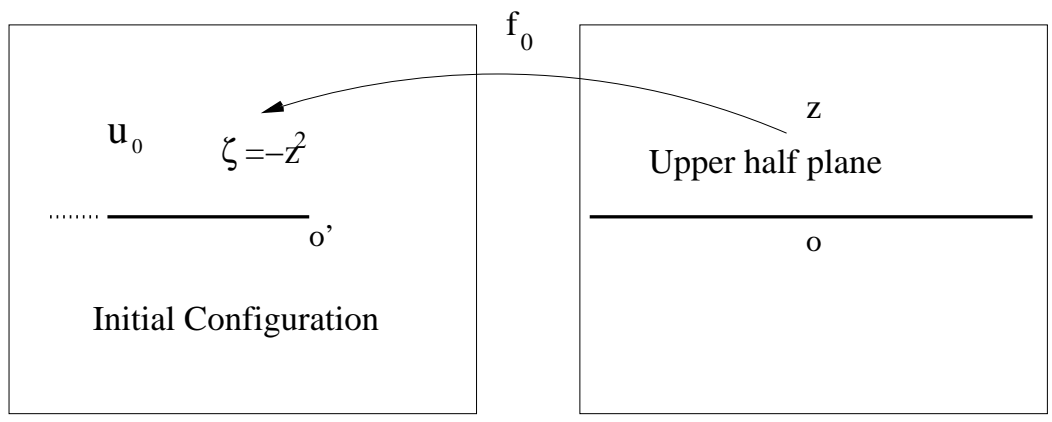

Figure 3: The map $f_{0}$.

may be extended analytically by symmetry to the whole plane, and then:

$$
h_{0}(z)=\sum_{n=0}^{\infty} c_{n} z^{n} \quad c_{n} \in \mathbb{R} .
$$

Therefore, $u_{0}$ admits the following convergent expansion:

$$
u_{0}=\operatorname{Re}\left[h_{0}\left(f_{0}^{-1}(\zeta)\right)\right]=c_{0}-c_{1} r^{1 / 2} \sin (\theta / 2)-c_{2} r \cos (\theta)+\ldots
$$

where

$$
\zeta=r e^{i \theta} \quad f_{0}^{-1}(\zeta)=\sqrt{-\zeta}
$$

We thus take (19) as the most general initial local field.

\subsection{Kinked configurations: conformal mapping}

For a given angle $\varphi$ we compute an expansion of the Energy released:

$$
f_{l}(z):=-(z-a(l))^{1-\alpha}(z-b(l))^{1+\alpha}, \quad \alpha:=\varphi / \pi(-1 \leq \alpha \leq 1)
$$

where:

$$
a(l):=-\sqrt{l}\left(\frac{1-\alpha}{1+\alpha}\right)^{\frac{1+\alpha}{2}}, \quad b(l):=\sqrt{l}\left(\frac{1+\alpha}{1-\alpha}\right)^{\frac{1-\alpha}{2}} .
$$

This map sends the upper half plane $\mathbb{H}$ onto the set $\mathbb{C} \backslash \Gamma_{l}$ as shown in Figure (4). It can be extended to the whole complex plane outside the interval $[-a(l), b(l)]$ and has the following properties:

$$
f_{l}(\mathbb{R})=\Gamma_{l} \quad \text { and } \quad f_{l}([a, b])=\Delta \Gamma
$$

Using the same arguments as before for $u_{l}=\operatorname{Re}\left[\eta_{l}(\zeta)\right]$ we can show that the complex function

$$
h_{l}(z):=\eta_{l}\left(f_{l}(z)\right)
$$


is analytic in the whole $\mathbb{C}$ and therefore can be expanded in powers of $z$ :

$$
h_{l}(z)=\sum_{n=0}^{\infty} c_{n}(l) z^{n}, \quad c_{n}(l) \in \mathbb{R} .
$$

We conclude that $u_{l}$ admits the following expansion in terms of some real coefficients $c_{n}(l)$ :

$$
u_{l}=\operatorname{Re}\left[\sum_{n=0}^{\infty} c_{n}(l)\left(f_{l}^{-1}(\zeta)\right)^{n}\right], \quad \zeta \in \mathbb{C} \backslash \Gamma_{l} .
$$

\subsection{Relationship between $c_{n}$ and $c_{n}(l)$}

Using analyticity, Neumann boundary conditions and the asymptotic matching condition at infinity we can show that

$$
c_{n}(l)=c_{n}+(n+1) c_{n+1} b_{0}(l)+O(l),
$$

where $b_{0}(l)=-\frac{1}{2}((1+\alpha) b(l)+(1-\alpha) a(l))$ is obtained from the expansion at infinity of the conformal map:

$$
\begin{aligned}
F_{l}(z) & =f_{0}^{-1} \circ f_{l}(z)=\sqrt{(z-a(l))^{1-\alpha}(z-b(l))^{1+\alpha}} \\
& =z+b_{0}(l)+\frac{b_{1}(l)}{z}+\frac{b_{2}(l)}{z^{2}} \ldots \quad z \rightarrow \infty .
\end{aligned}
$$

Notice that for $\alpha=0$ we have that $a(l)=-b(l)$ and then $b_{0}(l)=0$.

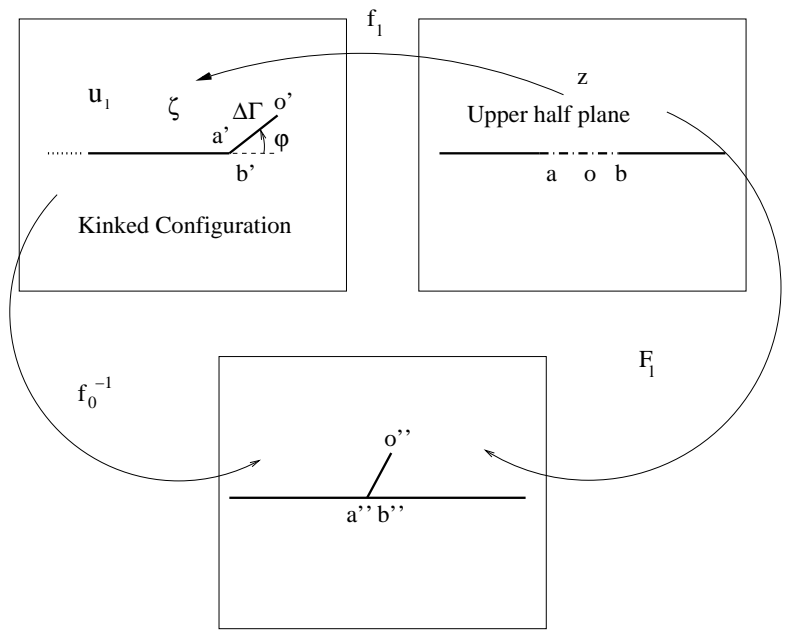

Figure 4: $f_{0}^{-1}, f_{l}$ and $F_{l}$ 
On the other hand, $F_{l}$ has the following scale invariance:

$$
F_{l}(z)=\sqrt{l} F_{1}(z / \sqrt{l}) \Rightarrow b_{n}(l)=\sqrt{l} b_{n}(1)
$$

and may be extended to the lower half plane, being a sectionally holomorphic function in $\mathbb{C} \backslash[a, b]$.

\subsection{Complex formula for the energy released}

An important tool in [14] is the energy formula

$$
\Delta E=\frac{\mu}{4 i} \int_{C} h_{l}^{\prime}(z) h_{0}\left(F_{l}(z)\right) d z,
$$

where $C$ is any simple closed curve surrounding the interval $[a, b]$ in the complex plane. By the analytic properties of $h_{l}, h_{0}$, and the expansion for $F_{l}$ we have that:

$$
\Delta E=\frac{\mu}{4 i} \int_{C}\left(\sum_{j=1} l^{\frac{j}{2}} j c_{j}(l) z^{j-1}\right)\left(\sum_{k=0} l^{\frac{k}{2}} c_{k}\left(F_{1}(z)\right)^{k}\right) d z,
$$

where $C$ encloses the interval $[a(1), b(1)]$.

The first term of (26) is given by

$$
-\Delta E=l \frac{\mu \pi}{4} c_{1}^{2}\left(\frac{1-\alpha}{1+\alpha}\right)^{\alpha}+O\left(l^{3 / 2}\right) .
$$

This provides a formula for the energy release rate in terms of the kinking angle:

$$
G(\alpha)=\lim _{l \rightarrow 0}\left(-\frac{\Delta E}{l}\right)=\frac{\mu \pi}{4} c_{1}^{2} A(\alpha) .
$$

This is the same behavior as that in (12), already obtained by Sih in [17] for uniform stresses at infinity. We can see that even if we take a general equilibrium field around the tip, it is not possible to obtain the crack deviation from the initial configuration by using the first order expansion for the energy released. Therefore, we must look at the energy functional in more detail.

\subsection{One more term in the expansion}

Using the complex formula for the energy released, the first two terms in the expansion are given by

$$
-\Delta E(\alpha)=\frac{\mu \pi}{4} c_{1}^{2} A(\alpha) l-\frac{4 \mu \pi}{3} c_{1} c_{2} B(\alpha) l^{\frac{3}{2}}+O\left(l^{2}\right)
$$






Figure 5: Two terms in the Energy expansion: the optimal angle.

where

$$
B(\alpha):=\frac{\alpha A(\alpha)^{\frac{3}{2}}}{\sqrt{1-\alpha^{2}}} .
$$

If we compute the maximum of $-\Delta E$ in terms of $\alpha$ for a fixed $l$, the optimal angle has the following asymptotic behaviour for $l \rightarrow 0$ :

$$
\alpha_{\max }=-\frac{4}{3} \frac{c_{2}}{c_{1}} l^{1 / 2}+o\left(l^{1 / 2}\right) .
$$

This suggests the following initial shape for $c_{2} \neq 0$ :

$$
x_{2} \approx-\frac{4 \pi}{3} \frac{c_{2}}{c_{1}}\left(x_{1}\right)^{3 / 2}, \quad x_{1}>0 .
$$

The contribution of the extra term introduces a singularity in crack curvature at that point. There is an interesting consequence of the analysis: the term of order $l^{3 / 2}$ in (27) has no influence on the total amount of energy released by the body for a finite extension of the crack, but it has a strong influence on crack geometry.

It has been recently pointed out to me $([1])$ that there is an interesting connection between this fact and some properties of the minimisers of the so-called Mumford-Shah functional [12]. The behaviour (29) is heuristically proposed as a singular energy minimiser when the tip is located at $x_{1}=0$ (see [12, Section 3]). The problems are not exactly the same, since for Mumford-Shah this cuspidal crack tip appears at the end of the curve and in our problem the singularity occurs between the initial crack tip and the optimal extension. Nevertheless, it seems that there is a close relationship between the optimal shapes for both problems. 


\subsection{Understanding the outcome for straight kinks}

Angle growing preference should be selected in such a way that the body is able to release the maximum amount of energy, provided the cost of bond breaking is the same in all directions. An intuitive way to look at our result is to check that the crack opening is larger in the direction defined by (28). Let us take first $c_{1}=1, c_{2}=0$, that is, a purely antisymmetric field. The comparison for different angles in the left of Figure 6 shows the preference of the $\alpha=0$ direction when $l=0.1$. On the other hand, if we add a symmetric contribution to the initial field with $c_{2}=1$, we obtain the pattern for the opening displacement shown on the right side of Figure 6.

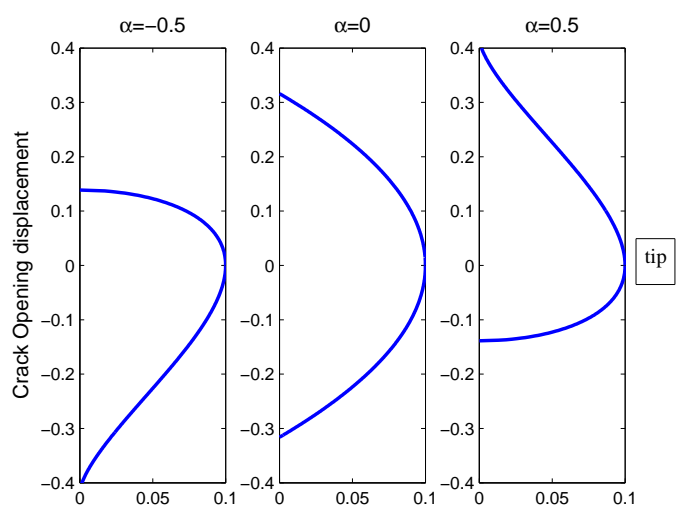

(a) $c_{2}=0$

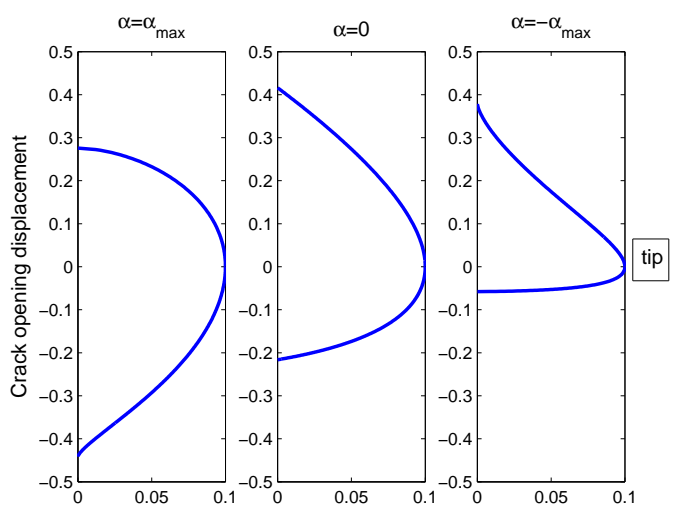

(b) $c_{2}=1$

Figure 6: Crack opening displacement.

Notice that for $\alpha=-\alpha_{\max }$ the opening is the lower one. On the other hand, the direction given in (28) is optimal among the three for maximum opening. The field was computed analytically, using the conformal map (20), the values $c_{1}=c_{2}=1$ for the initial field, $l=0.1$ and $c_{1}(l)=c_{1}+2 b_{0}(l) c_{2}$ for the expanded crack field (cf. (23)).

\section{Arbitrary extensions as virtual paths}

Kinked configurations are useful to quantify the deviation when we allow the crack to perform a sudden jump of length $l$. Nevertheless, the results show that this kind of abrupt change in direction is not admissible if the crack extends continuously; for vanishing lengths the optimal angle deviation must then go to zero. In this Section we will use the limit shape suggested by (29) to construct suitable trial paths in order to derive more precise conditions for crack stability. The technical details can be followed in [15]. 


\subsection{Loewner evolutions}

It is possible to generate the shape $x^{3 / 2}$ by means of a suitable conformal transform using the chordal Loewner equation for slit maps on the upper half plane (see [11]):

$$
\partial_{t} F_{t}(z)=-\frac{2 \partial_{z} F_{t}(z)}{z-\xi_{t}}, \quad F_{0}(z)=z
$$

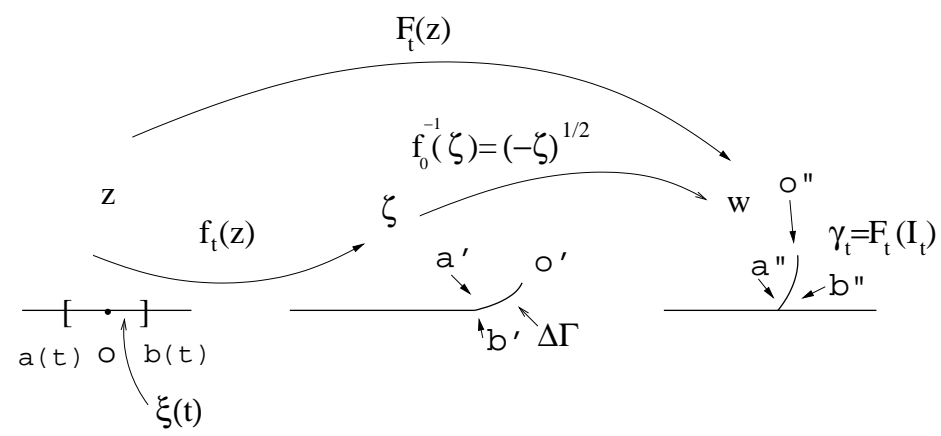

Figure 7: The conformal map $F_{t}$

Let us assume that $f_{0}^{-1}(\Delta \Gamma)$ is parametrised in a one to one way by $\gamma: t \mapsto \mathbb{H}$. We denote the evolution of the tip as $\gamma(t)$ and the whole set of the crack extension up to time $t$ by $\gamma_{t}$. By (30), it can be shown that each map $F_{t}: \mathbb{H} \mapsto \mathbb{H} \backslash \gamma_{t}$ is normalized as follows:

$$
F_{t}(z)=z-\frac{2 t}{z}+\frac{b_{2}(t)}{z^{2}}+O\left(1 / z^{3}\right)
$$

That is, for Loewner evolutions we always have that:

$$
b_{0}(t)=0, \quad b_{1}(t)=-2 t .
$$

We define $\xi_{t}:=F_{t}^{-1}(\gamma(t))$ (see Figure 7). Notice that $\xi_{t} \in \mathbb{R}$ and the parameter $t$ is not in general identified with crack length.

\subsection{Generating the virtual path}

Recent results of W. Kager, B. Nienhuis and L. Kadanoff [9] provide explicit solutions of the Loewner equation for some behaviours of $\xi_{t}$. For instance, taking $\xi_{t}=\lambda t,(30)$ takes the form

$$
\partial_{t} F_{t}(z)=-\frac{2 \partial_{z} F_{t}(z)}{z-\lambda t}, \quad F_{0}(z)=z
$$


This case is solved explicitly and it can be shown that generates the following path in the upper half plane:

$$
\gamma_{\lambda, t}=2 i t^{1 / 2}+\frac{2}{3} \lambda t-\frac{i}{18} \lambda^{2} t^{3 / 2}+O\left(t^{2}\right)
$$

The asymptotic behaviour of the tip on the physical plane is given by:

$$
x_{2}=-\frac{\lambda}{3} x_{1}^{3 / 2}+o\left(x_{1}^{3 / 2}\right) .
$$

Moreover, the coefficient $b_{2}(t)$ of this map is given by:

$$
b_{2}(t)=-\lambda t^{2}
$$

\subsection{The Energy Released Functional and the Anti-Symmetry Principle}

It is possible to write the following expansion for the energy released $(\mu=1)$ :

$$
\begin{aligned}
\Delta E(t) & =\frac{1}{4 i} \int_{C}\left(c_{1}^{2} F_{t}(z)+c_{1} c_{2}\left(F_{t}(z)^{2}+2 F_{t}(z)\left(z+b_{0}(t)\right)\right)\right) d z+O\left(\left|I_{t}\right|^{4}\right) \\
& =\frac{\pi}{2}\left(c_{1}^{2} b_{1}(t)+4 c_{1} c_{2}\left(b_{0}(t) b_{1}(t)+b_{2}(t)\right)\right)+O\left(\left|I_{t}\right|^{4}\right), \quad \text { as }\left|I_{t}\right| \rightarrow 0 .
\end{aligned}
$$

where $\left|I_{t}\right|$ is the length of the interval $I_{t}:=[a(t), b(t)]$ (see Figure 7 above ). Thus, taking into account (31), the asymptotic expression for the energy is given by:

$$
\Delta E(t)=\frac{\pi}{2}\left(-2 t c_{1}^{2}+4 c_{1} c_{2} b_{2}(t)\right)+O\left(\left|I_{t}\right|^{4}\right), \quad \text { as }\left|I_{t}\right| \rightarrow 0 .
$$

We have now all the ingredients to compute the energy released by the slit generated by (32). Inserting (34) in (36) we find that an extension evolving up to "time" $t$ will release an amount of energy given by

$$
\Delta E=-\pi c_{1}^{2} t-2 \pi \lambda c_{1} c_{2} t^{2}+O\left(t^{3}\right) .
$$

According to (4), in order to check the stability we must take into account the dissipation that is required to open this path. For this purpose we need the evolution of the length of the extended curve. The trial path in the physical plane is given by

$$
-\left(\gamma_{\lambda}(t)\right)^{2}=4 t-\frac{8}{3} i \lambda t^{\frac{3}{2}}-\frac{2}{3} \lambda^{2} t^{2}+O\left(t^{5 / 2}\right) .
$$

The length of this crack extension is given by the expansion

$$
l(t)=4 t+\frac{2 \lambda^{2}}{3} t^{2}+O\left(t^{3}\right) \quad t \rightarrow 0 .
$$


We use the last computations in the following equation:

$$
\Delta E+\Delta Q=\left(4 \kappa-\pi c_{1}^{2}\right) t+\left(\frac{2 \kappa \lambda^{2}}{3}-2 \pi \lambda c_{1} c_{2}\right) t^{2}+O\left(t^{3}\right)
$$

The term of order $t$ contains the balance between energy release rate $G(0)$ and $\kappa$. It cancels out due to the critical growth equation (13) and we then have that: $\left|c_{1}\right|=\sqrt{\frac{4 \kappa}{\pi}}$ . To satisfy the stable growth condition (3) the term of order $t^{2}$ must be positive or zero. Computing the minimum with respect to the parameter $\lambda$ we obtain $\lambda=\frac{3 \pi c_{1} c_{2}}{2 \kappa}$, and for this value we have:

$$
\Delta E+\Delta Q=-\frac{3}{2} \frac{\pi^{2}}{\kappa} c_{1}^{2} c_{2}^{2} t^{2}+O\left(t^{3}\right)<0 \quad \text { for } t \rightarrow 0,
$$

then violating the stability condition (4).

Therefore, since $c_{1} \neq 0$, a second necessary condition for a stable configuration is the following:

$$
c_{2}=0 \text {. }
$$

This is equivalent to impose $k_{2}=0$ in the typical expansion of the initial displacement field around the tip:

$$
u_{0}=k_{0}+k_{1} r^{1 / 2} \sin (\theta / 2)+k_{2} r \cos (\theta)+\ldots
$$

This condition cancels the symmetric contribution to the displacement near the tip, keeping the first (anti-symmetric) term of the expansion. We can state that (38) must hold on every stage of the propagation process, thus providing the second scalar condition to complete the free boundary problem. On the other hand, it has to be satisfied by other models imposing global minimization of the total energy (see for instance $[2,6]$ ).

\section{The case of a finite domain.}

The stability of the configuration was studied by imposing a Dirichlet condition at infinity (see (17)). It is natural to ask if the same outcome can be obtained on a finite domain. In other words, what happens if we freeze the displacements at a finite distance of the tip instead of fixing them at infinity? Bearing this question in mind, we will explain which parts of the previous discussion should be revised. This part of the work was not previously considered in the reviewed articles [14, 15].

\subsection{A simple setting for the boundary value problem}

We start with an initially straight crack configuration with the tip at the center of a circle of unit radius (the results are in fact independent of this particular geometry). The selected 
field satisfies the equilibrium equation (14), the Neumann boundary condition (15) and the finite energy condition near the tip (16). We now can apply a one-to-one conformal map $g_{0}$ transforming the upper half unit disc onto the initial domain. Take $u_{0}(x, y)$, the real part of an analytic function $\eta_{0}(x+i y)$ in $D \backslash \Gamma_{0}$, where

$$
D:=\{z:|z|<1\} .
$$

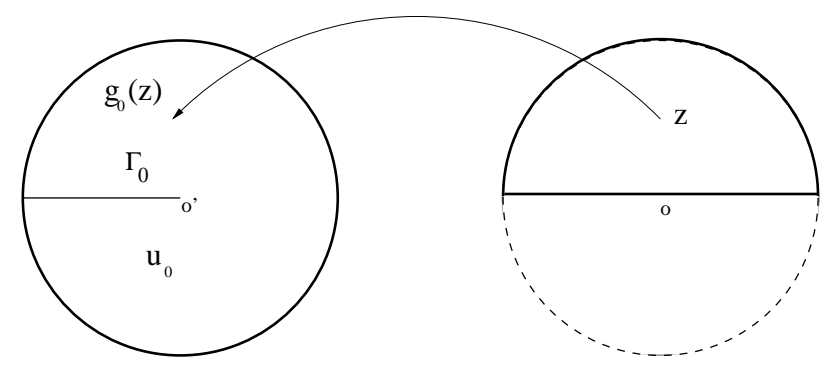

Figure 8: The basic conformal map $g_{0}$.

The analytic function $h_{0}:=\eta_{0} \circ g_{0}$ (defined on the right side of Figure 8) is extended by symmetry to the lower half disk due to the Neumman homogeneous condition. It then has a Taylor expansion with real coefficients as follows:

$$
u_{0}\left(g_{0}(z)\right)=\operatorname{Re}\left(h_{0}(z)\right)=\operatorname{Re}\left(\sum_{n=0}^{n} c_{n}(0) z^{n}\right) \quad c_{n} \in \mathbb{R} .
$$

For simplicity, we will assume that (39) is convergent on some open set containing $\bar{D}$.

We define the field $u_{t}$ as the one satisfying the equilibrium equation $\Delta u_{t}=0$ on $D \backslash \Gamma_{t}$, the Neumann boundary condition $\partial u / \partial n=0$ on $\Gamma_{t}$, and the Dirichlet boundary condition on the unit circle:

$$
u_{t}(x, y)=u_{0}(x, y) \quad \text { for }|x+i y|=1 .
$$

There is an analytic function $\eta_{t}$ in $D \backslash \Gamma_{t}$ such that $u_{t}(x, y)=\operatorname{Re} \eta_{t}(x+i y)$ and the function

$$
h_{t}(z):=\eta_{t}\left(g_{t}(z)\right)
$$

is analytic in the upper half unit disk and can be extended to the whole disk by the Neumann homogeneous condition. The map $g_{t}(z)$ sends the upper half unit disk to $D \backslash \Gamma_{t}$ as shown in Figure 9. We have that $h_{t}$ admits the following expansion:

$$
h_{t}(z)=\sum_{n=0}^{\infty} c_{n}(t) z^{n} \quad c_{n}(t) \in \mathbb{R} .
$$




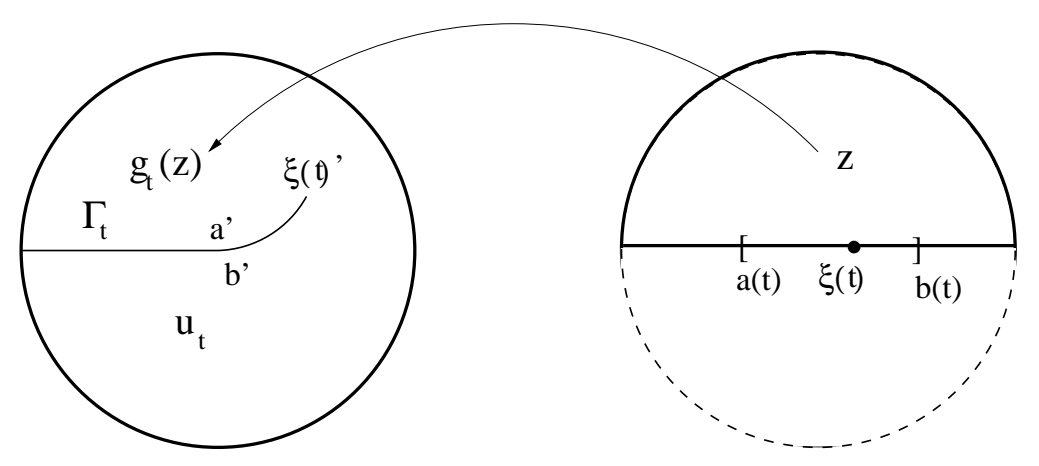

Figure 9: The basic map $g_{t}$.

The main difference with the case of an unbounded domain corresponds to the behaviour of the coefficients $c_{n}(t)$ for $t \rightarrow 0$. The Dirichlet condition is applied on a curve at a finite distance of the tip, while in the former case it was imposed on a unique point at infinity.

Assuming for a moment that we know $g_{t}(z)$, we can write Schwartz integral representation of $h_{t}(z)$ for the unit disk. The boundary values of the real part of $h_{t}(z)$ are obtained from the following chain of equalities:

$$
\begin{aligned}
u_{t}\left(g_{t}\left(e^{i \theta}\right)\right) & =u_{0}\left(g_{t}\left(e^{i \theta}\right)\right) \quad\left(\left|g_{t}\left(e^{i \theta}\right)\right|=1 \text { cf. Figure } 9\right), \\
u_{0}\left(g_{t}\left(e^{i \theta}\right)\right) & =u_{0}\left(g_{0}\left(g_{0}^{-1} \circ g_{t}\left(e^{i \theta}\right)\right)\right) \\
u_{0}\left(g_{0}\left(G_{t}\left(e^{i \theta}\right)\right)\right) & =\operatorname{Re}\left\{h_{0}\left(G_{t}\left(e^{i \theta}\right)\right)\right\}
\end{aligned}
$$

where $G_{t}$ is defined as

$$
G_{t}:=g_{0}^{-1} \circ g_{t}
$$

This map is analytic on a circular ring whose interior circle contains the interval $[a(t), b(t)]$ (cf. Figure 10). It admits a Laurent expansion with real coefficients that we write as follows:

$$
G_{t}(z)=G_{t}^{+}(z)+G_{t}^{-}(z)
$$

with

$$
G_{t}^{+}(z):=\sum_{k=0}^{\infty} a_{k}(t) z^{k} \quad G_{t}^{-}(z):=\sum_{k=1}^{\infty} b_{k}(t) z^{-k} .
$$

Notice that $G_{t}(z) \rightarrow z$ and $b_{k+1}(t) \ll b_{k}(t)$ for $t \rightarrow 0$. This last assertion is a consequence of the Area Theorem (see the Appendix). Notice that $G_{t}^{-}$is analytic outside of a vanishing circle containing $[a(t), b(t)]$.

To simplify a bit the notation we define the boundary values of the real part of $h_{t}$ as $U_{t}(\theta):=\operatorname{Re}\left\{h_{0}\left(G_{t}\left(e^{i \theta}\right)\right)\right\}$. Using Schwartz integral in the unit disk, we have the following 


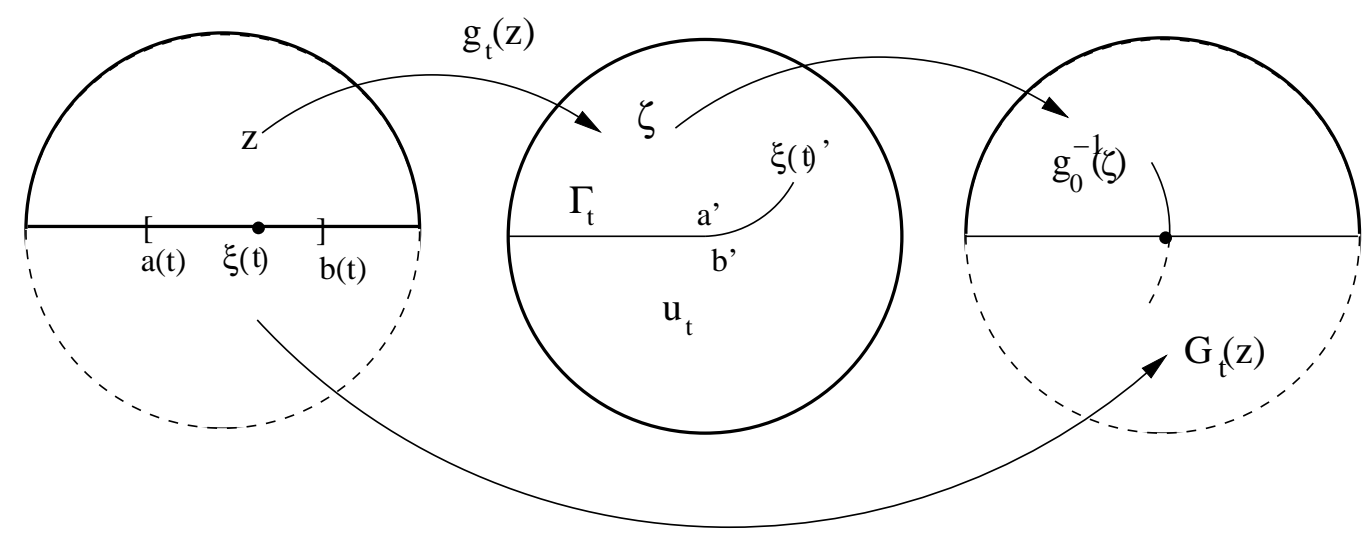

Figure 10: The mapping properties of the extended $G_{t}$.

explicit formula

$$
h_{t}(z)=\frac{1}{2 \pi} \int_{-\pi}^{\pi} \frac{e^{i \theta}+z}{e^{i \theta}-z} U_{t}(\theta) d \theta \underset{\zeta:=e^{i \theta}}{=} \frac{1}{2 \pi i} \int_{|\zeta|=1} \frac{\zeta+z}{\zeta-z} \operatorname{Re}\left\{h_{0}\left(G_{t}(\zeta)\right)\right\} \frac{d \zeta}{\zeta}
$$

We can then write (cf. (40)):

$$
h_{t}(z)=\sum_{n=0}^{\infty} c_{n}(t) z^{n}=(1+z) \sum_{n=0}^{\infty}\left(\frac{1}{2 \pi} \int_{-\pi}^{\pi} U_{t}(\theta) e^{-i n \theta} d \theta\right) z^{n}
$$

We want now to relate the coefficients $c_{n}(t)$ with the ones of the initial field $c_{n}(0)$. Using the expansion for $h_{0}(z)$ we have that:

$$
U_{t}(\theta)=\operatorname{Re}\left\{h_{0}\left(G_{t}\left(e^{i \theta}\right)\right)\right\}=\operatorname{Re}\left\{\sum_{k=0}^{\infty} c_{k}(0)\left(G_{t}\left(e^{i \theta}\right)\right)^{k}\right\}
$$

The expression for the first coefficient (notice that $U_{t}(-\theta)=U_{t}(\theta)$ ) should read (we drop for a while the dependence on $t$ of the $a_{i}$ 's and $b_{i}$ 's):

$$
\begin{aligned}
c_{0}(t) & =\operatorname{Re} \sum_{j=0}^{\infty} c_{k}(0)\left\{\frac{1}{\pi} \int_{0}^{\pi}\left(G_{t}\left(e^{i \theta}\right)\right)^{j}\right\} d \theta \\
& =c_{0}(0)+c_{1}(0) a_{0}+c_{2}(0)\left(a_{0}^{2}+2 a_{1} b_{1}+2 a_{2} b_{2}\right)+\ldots
\end{aligned}
$$

Similarly, we obtain that for $c_{1}(t)$,

$$
\begin{aligned}
c_{1}(t) & =\sum_{j=0}^{\infty} c_{j}(0) \frac{2}{\pi} \int_{0}^{\pi} \operatorname{Re}\left(G_{t}\left(e^{i \theta}\right)\right)^{j} \cos (\theta) d \theta \\
& =c_{1}(0)\left(a_{1}+b_{1}\right)+2 c_{2}(0)\left(a_{0}\left(a_{1}+b_{1}\right)+a_{1} b_{2}+a_{2}\left(b_{1}+b_{3}\right)\right)+\ldots
\end{aligned}
$$


For $c_{2}(t)$ we have:

$$
\begin{aligned}
c_{2}(t) & =\sum_{j=0}^{\infty} c_{j}(0) \frac{2}{\pi} \int_{0}^{\pi} \operatorname{Re}\left(G_{t}\left(e^{i \theta}\right)\right)^{j} \cos (2 \theta) d \theta \\
& =c_{1}(0)\left(a_{2}+b_{2}\right)+c_{2}(0)\left(a_{1}^{2}+2 a_{0} b_{2}+2 a_{1} b_{3}\right)+\ldots
\end{aligned}
$$

the main contribution being (notice that $a_{1}(t) \rightarrow 1$ for $t \rightarrow 0$ ):

$$
c_{2}(t)=c_{2}(0) a_{1}^{2}(t)+o(1) .
$$

We can now apply the complex version of the energy release formula (cf. 25):

$$
\Delta E=\frac{1}{4 i} \int_{C} h_{t}^{\prime}(z) h_{0}\left(G_{t}(z)\right) d z=\frac{1}{4 i} \int_{C}\left(\sum_{k=1}^{\infty} k c_{k}(t) z^{k-1}\right)\left(\sum_{j=0}^{\infty} c_{j}(0)\left(G_{t}(z)\right)^{j}\right) d z
$$

where $C$ is a simple curve enclosing the interval $[a(t), b(t)]$ and contained in $D$. Taking into account $(45,46)$ we can approximate the expression for $\Delta E$ as follows:

$$
\begin{aligned}
\Delta E \approx & \frac{\pi}{2}\left\{c_{1}(t)\left(c_{1}(0) b_{1}(t)+2 c_{2}(0)\left(a_{0}(t) b_{1}(t)+a_{1}(t) b_{2}(t)\right)\right)\right. \\
& \left.+2 c_{2}(t)\left(c_{1}(0) b_{2}(t)+2 c_{2}(0)\left(a_{0}(t) b_{2}(t)+a_{1}(t) b_{3}(t)\right)+\ldots\right)\right\} \\
\approx & \frac{\pi}{2}\left(c_{1}^{2}(0) a_{1}(t) b_{1}(t)+2 c_{1}(0) c_{2}(0)\left(a_{0}(t) a_{1}(t) b_{1}(t)+a_{1}^{2}(t) b_{2}(t)+a_{0}(t) b_{1}^{2}(t)\right) \cdots+\right. \\
& \left.2 c_{1}(0) c_{2}(0) a_{1}^{2}(t) b_{2}(t)+\ldots\right)
\end{aligned}
$$

Summing up:

$$
\Delta E \approx \frac{\pi}{2}\left(c_{1}^{2}(0) a_{1}(t) b_{1}(t)+2 c_{1}(0) c_{2}(0)\left(a_{0}(t) a_{1}(t) b_{1}(t)+2 a_{1}^{2}(t) b_{2}(t)+a_{0}(t) b_{1}^{2}(t)\right)\right.
$$

Notice that for $a_{0}=0$ and $a_{1}=1$ we obtain the same asymptotic expression as that derived for the unbounded domain (cf. (35)).

\subsection{The construction of the conformal map}

To justify the anti-symmetry principle for a finite domain it only remains to show that a suitable conformal map can be constructed, and that its coefficients $a_{0}(t), a_{1}(t), b_{1}(t), b_{2}(t)$ produce the instability given in (37). We will proceed by using a modified map of that obtained from the Loewner equation in Section 4.2.

Let us take firstly the map $F_{t}$ for the upper half plane, generated with (32). By restricting the domain, we can see that $F_{t}$ sends the unit circle with a cut on the real line to a nearly circular domain for small $t$ with a cut inside (cf. Figure 11). A slight modification of the map $F_{t}$ should carry us to the desired $G_{t}$. This change will be applied as follows: 
1. We map the boundary of the unit disk $\partial D$ to the boundary of a nearly circular domain by means of $F_{t}(z)$.

2. We consider the holomorphic map $\omega_{t}(z)$ that carries the region bounded by $F_{t}(\partial D)$ to the unit disc with $\omega_{t}(0)=0$ and $\omega_{t}(z)=\overline{\omega_{t}(\bar{z})}$. $G_{t}(z)$ will be given by the composition $\omega_{t} \circ F_{t}(z)$.

We summarize in the following Figure the properties of the modified $F_{t}$.



Figure 11: The map $\omega_{t}$ modifying $F_{t}$.

We now follow [13] for the technical details and define the real function

$$
\rho(\theta):=\lim _{t \rightarrow 0} \frac{\left|F_{t}\left(e^{i \theta}\right)\right|-1}{t} .
$$

Thus, $r=1+t \rho(\theta)+o(t)$ is the polar equation for the boundary of the modified domain (the up-right domain in Figure 11). Notice that $\rho(\theta)=\rho(-\theta)$ due to the symmetry properties of the map $F_{t}$. We now have an explicit formula for the first terms of $\omega_{t}$ (cf. [13, p. 264]):

$$
\omega_{t}(z)=z\left(1-\frac{t}{2 \pi} \int_{-\pi}^{\pi} \frac{e^{i \theta}+z}{e^{i \theta}-z} \rho(\theta) d \theta+o(t)\right) .
$$

We may write this as follows:

$$
\omega_{t}(z)=z(1-t \psi(z)+o(t)), \quad \psi(z)=\sum_{n=0}^{\infty} p_{n} z^{n} .
$$


We now look at $G_{t}(z)$ :

$$
G_{t}(z)=\omega_{t}\left(F_{t}(z)\right)=F_{t}(z)\left(1-t \psi\left(F_{t}(z)\right)+o(t)\right),
$$

and then we can write

$$
G_{t}(z)=\left(1-t p_{0}\right) F_{t}(z)-t\left(p_{1} F_{t}^{2}+p_{2} F_{t}^{3}+\ldots\right)
$$

Let us denote by $\tilde{b}_{n}(t)$ the coefficients of the expansion of $F_{t}(z)$ (cf. 24) to distinguish them from the ones of $G_{t}(z)$ (cf. 41). We have that:

$$
\begin{aligned}
a_{0}(t) & =\left(1-t p_{0}\right) \tilde{b}_{0}(t)-t\left(p_{1}\left(\tilde{b}_{0}^{2}(t)+2 \tilde{b}_{1}(t)\right)\right)+o\left(t \tilde{b}_{1}(t)\right) \\
a_{1}(t) & =\left(1-t p_{0}\right)-t\left(2 p_{1} \tilde{b}_{0}(t)+3 p_{2} \tilde{b}_{1}(t)\right)+o(t) \\
b_{1}(t) & =\left(1-t p_{0}\right) \tilde{b}_{1}(t)-t\left(2 p_{1}\left(\tilde{b}_{0}(t) \tilde{b}_{1}(t)+\tilde{b}_{2}(t)\right)+3 p_{2}\left(\tilde{b}_{0}^{2} \tilde{b}_{1}+\tilde{b}_{1}^{2}+\tilde{b}_{0} \tilde{b}_{2}\right)\right)+O\left(t \tilde{b}_{1}\right) \\
b_{2}(t) & =\left(1-t p_{0}\right) \tilde{b}_{2}(t)-t\left(2 p_{1}(t) \tilde{b}_{3}(t)\right)+o\left(t^{2}\right)
\end{aligned}
$$

For the linear forcing path generated by Loewner's equation we have that

$$
\tilde{b}_{0}(t)=0, \quad \tilde{b}_{1}(t)=-2 t, \quad \tilde{b}_{2}=-\lambda t^{2} .
$$

Then there holds:

$a_{0}(t)=4 p_{1} t^{2}+o\left(t^{2}\right), \quad a_{1}(t)=\left(1-t p_{0}\right)+o(t), \quad b_{1}(t)=-2 t\left(1-t p_{0}\right)+o\left(t^{2}\right), \quad b_{2}(t)=-\lambda t^{2}+o\left(t^{2}\right)$.

The energy released can be written now as (cf. 47):

$$
\Delta E \approx-\frac{\pi}{2}\left(1-t p_{0}\right)^{2}\left(2 c_{1}^{2}(0) t+4 \lambda c_{1}(0) c_{2}(0) t^{2}\right)+o\left(t^{2}\right)
$$

Notice that this expression is (up to order $t^{2}$ ) the one obtained for the unbounded domain multiplied by the factor $\left(1-t p_{0}\right)^{2}$. This factor is non-universal in the sense that $p_{0}$ depends on the geometric properties of the body boundary:

$$
p_{0}=\frac{1}{\pi} \int_{0}^{\pi} \rho(\theta) d \theta
$$

This is consistent with the asymptotics of the stress intensity factor obtained by Leblond in [10], but a thorough discussion of this subject would carry us far from our main point here. We mention by pass that the physical length of the crack is (up to order $t$ ) $l \sim 4 t$, and then the energy release rate is consistent with the expression obtained before:

$$
\lim _{t \rightarrow 0}-\frac{\Delta E}{4 t}=\frac{\pi}{4} c_{1}^{2}(0)
$$


This is also in accordance to Leblond's paper, since we should not have an explicit dependence of the body geometry in this order (of course, there is an implicit information encoded in $c_{1}$ ).

To conclude, it remains to find the expression for the length in terms of $t$, up to order $t^{2}$. We have to take into account that $\omega_{t}(z) \approx\left(1-t p_{0}\right) z$. The path in the (non physical) lower domain in Figure 11 is given by (cf. 33):

$$
\gamma_{\lambda, t}=\left(1-t p_{0}\right)\left(2 i t^{1 / 2}+\frac{2}{3} \lambda t-\frac{i}{18} \lambda^{2} t^{3 / 2}\right)+O\left(t^{2}\right)
$$

In the physical domain we should have, recalling that $G_{t}(z)=g_{0}^{-1} \circ g_{t}(z)$ and $g_{0}(z)=-z^{2}$ :

$$
g_{0}\left(\gamma_{\lambda, t}\right)=\left(1-t p_{0}\right)^{2}\left(4 t-\frac{8}{3} i \lambda t^{\frac{3}{2}}-\frac{2}{3} \lambda^{2} t^{2}+O\left(t^{5 / 2}\right)\right)
$$

Therefore, the length of the path is given by:

$$
l(t)=\left(1-t p_{0}\right)^{2}\left(4 t+\frac{2 \lambda^{2}}{3} t^{2}\right)+O\left(t^{3}\right) \quad t \rightarrow 0
$$

We conclude that the dissipation term obtained in the unbounded case is to be multiplied by the positive non-universal factor $\left(1-t p_{0}\right)^{2}$, the same as that multiplying the relevant

contribution to the released energy in (49). Therefore, selecting $\lambda=\frac{3 \pi c_{1} c_{2}}{2 \kappa}$ as in the unbounded case, the constructed virtual path shows again the unstable character of an initial field with $c_{2}(0) \neq 0(\mathrm{cf} .(37))$ :

$$
\Delta E+\Delta Q=-\left(1-t p_{0}\right)^{2}\left(\frac{3}{2} \frac{\pi^{2}}{\kappa} c_{1}^{2} c_{2}^{2} t^{2}\right)+O\left(t^{3}\right)<0 \quad \text { for } t \rightarrow 0
$$

This path violates the stability assumption (4) and provides a necessary condition for the initial field, namely $c_{2}=0$.

\section{Acknowledgements}

This research was supported by Spanish research project BFM 2004-05634. Part of this work was presented at the International Symposium on Defects and Material Mechanics held in Aussois, 25-29 March 2007. I wish to acknowledge the organizers C. Dascalu and G. Maugin for the kind invitation to participate in this meeting.

\section{References}

[1] M. Buliga, private communication. 
[2] M. Buliga, "Energy minimizing brittle crack propagation", Journal of Elasticity, 52 (1999), pp. 201-238.

[3] B. Cotterell and J.R. Rice, "Slightly curved or kinked cracks", International Journal of Fracture, 16, No. 2 (1980), pp. 155-169.

[4] P.L. Duren (1983). Univalent Functions, A Series of Comprehensive Studies in Mathematics: 259, Springer-Verlag, New York.

[5] G.R. Irwin, "Analysis of stresses and strains near the end of a crack transversing a plate", Journal of Applied Mechanics 24 (1957), pp. 361-364.

[6] G.A. Francfort and J.J. Marigo (1998), "Revisiting brittle fracture as an energy minimization problem", Journal of the Mechanics and Physics of Solids, Vol. 46, No.8, pp. 1319-1342.

[7] R.V. Goldstein and R.L. Salganik, "Brittle fracture of solids with arbitrary cracks", International Journal of Fracture, 10 (1974), pp. 507.

[8] M. Gurtin, "On the energy release rate in quasi-static elastic crack propagation", Journal of Elasticity, Vol. 9, No 2, (April 1979), 187-195.

[9] W. Kager, B. Nienhuis and L. Kadanoff (2004) "Exact Solutions for Loewner Evolutions", Journal of Statistical Physics, Vol. 115, Nos.3/4, May 2004, pp. 805-822.

[10] J.B.Leblond (1989), "Crack paths in plane situations I: General form of the expansion of the stress intensity factors", Int. J. Solids Structures Vol. 25, No. 11, pp. 1311-1325.

[11] D.E.Marshall and S.Rohde (2001) "The Loewner differential equation and slit mappings", J. Amer. Math. Soc. 18 (2005), 763-768.

[12] D. Mumford, J. Shah, "Optimal Approximations by Piecewise Smooth Functions and Associated Variational Problems", Communications on Pure and Applied Mathematics (1989), Vol. XLII, pp 577-685.

[13] Z. Nehari (1975), Conformal Mapping, Dover Publications New York.

[14] G.E.Oleaga, "On the path of a quasi-static crack in Mode III", Journal of Elasticity, 76, nr.2 (August 2004), pp 163-189.

[15] G.E.Oleaga, "The classical theory of Univalent Functions and Quasi-static Crack Propagation", European Journal of Applied Mathematics vol. 17, pp. 233-255, (2006) Cambridge University Press.

[16] J.R. Rice (1968). "Mathematical analysis in the mechanics of fracture", in Fracture, Vol. 2, ed. H. Liebowitz. New York: Academic, pp. 191-311. 
[17] G.C. Sih, "Stress distribution near internal crack tips for longitudinal shear problems", Journal of Applied Mechanics (march 1965), pp. 51-58.

\section{A The Area Theorem for the Laurent expansion}

Consider a family of univalent functions indexed by $t$, on a circular ring whose interior circle is of radius $\rho(t)$, with $\rho(t) \rightarrow 0$ as $t \rightarrow 0$. Moreover, assume that it admits a Laurent expansion with real coefficients written as (41). It is possible to show that the area inside the image circle $C_{r}$ of radius $r$ inside the circular ring, is given by:

$$
\text { Area }=\frac{1}{2} \operatorname{Im} \int_{C_{r}} \overline{G_{t}(z)} G_{t}^{\prime}(z) d z .
$$

Taking into account the Laurent expansion for $G_{t}$ in (41), we can write:

$$
\begin{aligned}
\text { Area } & =\frac{1}{2} \operatorname{Im} \int_{C_{r}} \overline{\left(\sum_{n=0}^{\infty} a_{n} z^{n}+\sum_{n=1}^{\infty} b_{n} z^{-n}\right)}\left(\sum_{n=1}^{\infty} n a_{n} z^{n-1}-\sum_{n=1}^{\infty} n b_{n} z^{-n-1}\right) d z \\
& =\pi\left(\sum_{n=1}^{\infty} n r^{n} a_{n}^{2}-\sum_{n=1}^{\infty} n r^{-n} b_{n}^{2}\right)
\end{aligned}
$$

where we avoided the dependence on $t$ for the coefficients. Since Area is non-negative, and taking $r \rightarrow \rho(t)$ we finally obtain the inequality:

$$
\sum_{n=1}^{\infty} n \rho(t)^{-n} b_{n}^{2} \leq \sum_{n=1}^{\infty} n \rho(t)^{n} a_{n}^{2} .
$$

The right hand side is convergent by construction; in fact, it goes to zero for $t \rightarrow 0$. Notice that

$$
\frac{1}{2} \operatorname{Im} \int_{C_{r}} \overline{G_{t}^{+}(z)} G_{t}^{+\prime}(z) d z=\pi \sum_{n=1}^{\infty} n \rho(t)^{n} a_{n}^{2} .
$$

We can then take the limit for $r \rightarrow \rho(t)$ since $G_{t}^{+}$is analytic inside the outer circle of the ring (cf. (42)). This shows that the coefficients of the left hand series in (50) must be, at least, bounded:

$$
n \rho(t)^{-n} b_{n}^{2}(t) \leq M \Rightarrow b_{n}^{2}(t) \leq \frac{M}{n} \rho(t)^{n}, \quad \text { for } n \geq 1
$$

for a suitable positive constant $M$. This provides the asymptotic relative magnitude of the different $b_{n}$ 's for $t \rightarrow 0$. 